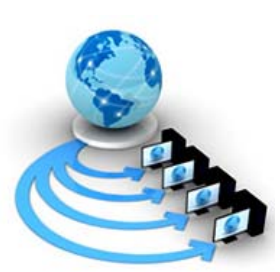

Volume 9, No. 1, January-February 2018

International Journal of Advanced Research in Computer Science

RESEARCH PAPER

\author{
Available Online at www.ijarcs.info
}

\title{
PERFORMANCE COMPARISON OF OBJECT ACQUISITION USING AUTOMATIC SCANNING AND FACIAL FEATURE TRACKING
}

\author{
Hari Singh \\ Research Scholar \\ IKG Punjab Technical University \\ Kapurthala (Punjab), India
}

\author{
Jaswinder Singh \\ Associate Professor \\ Beant College of Engineering and Technology \\ Gurdaspur (Punjab), India
}

\begin{abstract}
This paper presents a comparison of object acquisition in an HCI system using two different techniques: automatic scanning and facial feature tracking. In automatic scanning the focus moves from one object to next object automatically after a predefined time period called scanning time. Automatic scanning has been implemented by using MATLAB algorithm which virtually activates the tab key after each scanning time and the focus moves from object to object. The user activates a selection trigger for selection of the object when the focus comes over the object of interest. Whereas, in facial feature tracking approach the mouse cursor is moved in proportion to the movement of user's face. To implement this technique Camera Mouse has been used which requires a simple webcam. It continuously takes facial images of the user and finds the mouse cursor position from the face coordinates. The two techniques are compared based upon accuracy and acquisition time for acquisition of text and graphic objects.
\end{abstract}

Keywords: Object acquisition; HCI systems; automatic scanning; face tracking.

\section{INTRODUCTION}

A user controls computer with the help of an interface called as human computer interaction (HCI) system. The two important functions performed by an HCI system are object acquisition and object selection. Object acquisition refers to the movement of focus/cursor over the object to be selected and object selection means activation of a selection trigger when the focus/cursor comes over the desired object. Some of the techniques used for object acquisition are: eye gaze tracking [1], [2], face tracking [3], [4], facial feature tracking [5], scanning [6]-[9], and tongue movement [10]. Object selection can be performed by using key trigger [11], eye blinking [12], [13], dwell time trigger [14], [15], antisaccades, gaze gestures, on-off screen buttons, dashers, pEYEs [16], mouth opening click [17], tooth clicker [2], brows up clicker [8], EMG clicking [18], and clicking with smiling [19]. The major deciding factors for selection of a combination of object acquisition and selection techniques are: physical condition of a user, cost, performance, and ease of use.

This paper presents a comparison of performance of automatic scanning and face tracking techniques for object acquisition. From the overall comparison it is observed that the automatic scanning technique requires minimum physical effort to move the focus or mouse cursor. This technique is an obvious choice for persons suffering from motor neuron diseases because they have no/less control over their physical movements. The technique also offers a feature of automatic page scrolling while surfing html files or other type of files with multiple pages. The major concern with face tracking technique is the cursor stability, especially, when the objects of small size are to be acquired. Also, this technique requires face/head movements from the user and is difficult to be used for long time, especially, for disabled persons.

\section{METHODOLOGY}

For testing of performance of automatic scanning and facial feature tracking techniques an experiment was conducted for acquisition of text and graphic objects using both the techniques. The automatic scanning technique does not require any hardware whereas face tracking technique needs a webcam for acquisition of user facial images.

\section{A. Implementation of automatic scanning technique for focus movement}

In automatic scanning a MATLAB algorithm activates the tab key to move focus over objects placed on computer screen. The time taken by the algorithm to move focus from one object to next object is called as scanning time. The process of moving focus over objects using this technique is shown in figure 1 in the form of a flow chart. The scanning of objects starts from very first object placed in the file and reaches to the last object after scanning all the objects placed in between. The user activates a selection trigger, which is eye blink in this research, if the object having focus is the object to be selected; otherwise, the focus is transferred to the next object. In this way, the object of interest is selected with very less physical efforts and the user has to just blink his/her eye for final selection of an object.

\section{B. Facial feature tracking for cursor control}

In this technique the mouse cursor movement is controlled in proportion to the user face movement. To implement this technique 'Camera Mouse [5]' (Version 1.0 2016) (www.cameramouse.org/download.html) has been used which is a freely available software and allows the control of mouse cursor on a Windows computer using face movements. This technique is implemented by using a simple webcam and a windows computer. It is simple, easy to use and can be used under variable lighting conditions. 


\section{Experiment}

An experiment was conducted to check the performance of automatic scanning and camera mouse for object selection in which ten healthy users voluntarily participated. The users were 3 females and 7 males in the age group of 16 -43 years $($ mean $=32, \mathrm{SD}=9.0185)$. The participants were regular computer users and did not have experience of using camera mouse. A training session for the participants was conducted before starting the actual experiment and the users were briefed about the task to be performed. The users were asked to bring the focus/cursor over object of interest by using both the techniques. Two types of objects were considered for acquisition: text objects and graphic objects. The text objects were the hyperlinks of variable sizes in an html file and the graphic objects were the icons placed on the computer desktop.

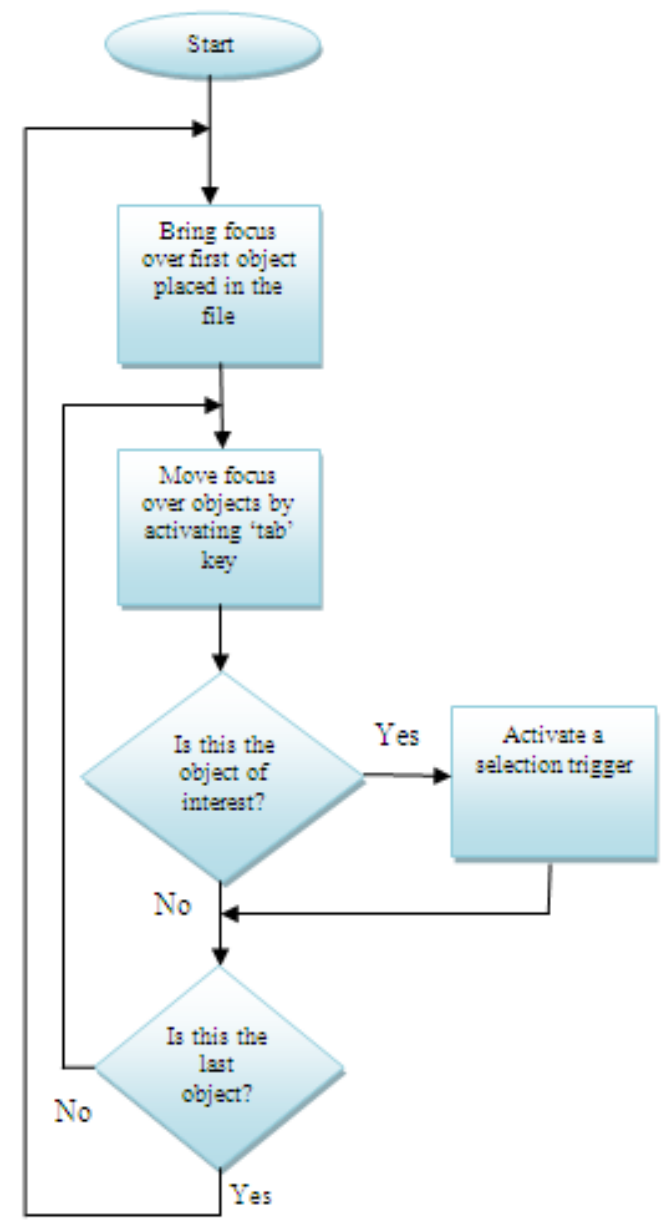

Figure 1. The process of object acquisition using automatic scanning

\section{RESULTS AND DISCUSSION}

As the scanning time is an important factor and decides the overall performance of the automatic scanning technique of object acquisition, therefore, in the initial phase of implementation of this technique a trial was conducted to find the appropriate value of scanning time which gives maximum accuracy. A graph in figure 2 shows acquisition time and accuracy of the automatic scanning technique for different values of scanning time $(0.5$ to $1.2 \mathrm{~s})$. It can be observed from the graph that acquisition time increases with increase in scanning time. The system accuracy increases from $62 \%$ to $88 \%$ as the scanning time increases from 0.5 to 1.0 seconds and becomes constant thereafter. Therefore, the next part of the experiment was performed by considering scanning time $=1.0 \mathrm{~s}$ for which the system has given average accuracy of $88 \%$.

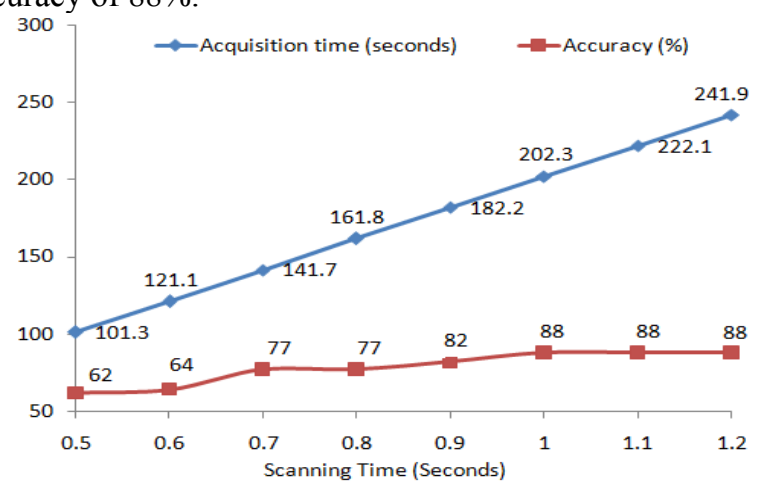

Figure 2. Automatic scanning technique performance for varying scanning time

In the main part of the experiment, performance analysis of automatic scanning technique and camera mouse was performed for acquisition of text and graphic object acquisition. Table 1 gives the performance results in the form of average accuracy and average scanning time for both the systems. A one-way ANOVA test reveals the automatic scanning technique gives significantly better accuracy $(F(1,18)=10.28919), p<0.01)$ for acquisition of text objects in an html file where page scrolling is required, but the difference in accuracy for selection of graphic objects is not significant $(\mathrm{F}(1,18)=2.922078, \mathrm{~ns})$. The difference in acquisition time is not significant for selection of both text $(\mathrm{F}(1,18)=0.153159, \mathrm{~ns})$ and graphic objects $(\mathrm{F}(1,14)=$ $1.176991, \mathrm{~ns})$.

\section{CONCLUSION}

The performance comparison of automatic scanning technique and facial feature tracking for acquisition of graphic and text objects is presented in this paper. Automatic scanning technique has been implemented by using MATLAB and facial features tracking has been performed by using Camera Mouse. From the overall comparison it has been observed that the automatic scanning technique gives significantly better accuracy for acquisition of graphic objects placed in an html file and the difference in accuracy for selection of graphic objects in not significant. Further, no significant difference has been observed in acquisition time for acquisition of graphic and text objects using both the techniques. The automatic scanning is easy to use because it requires minimum physical efforts to be made by the user for object acquisition and it also provides the feature of automatic page scrolling in a multiple pages file. 
Table I. Performance results of object acquisition using automatic scanning and facial feature tracking

\begin{tabular}{|c|c|c|c|}
\hline \multicolumn{2}{|c|}{} & Automatic Scanning & Facial Feature Tracking \\
\hline \multirow{2}{*}{ Accuracy (\%) } & Text Objects & 87 & 72 \\
\cline { 2 - 4 } & Graphic Objects & 91.2 & 97.5 \\
\hline $\begin{array}{c}\text { Acquisition Time } \\
\text { (s) }\end{array}$ & Text Objects & 202.3 & 159 \\
\cline { 2 - 4 } & Graphic Objects & 16.5 & 11.8 \\
\hline
\end{tabular}

\section{REFERENCES}

[1] V. N. Hegde, R. S. Ullagaddimath, and K. S, "Low Cost Eye Based Human Computer Interface System," in 2016 IEEE Annual India Conference (INDICON), 2016.

[2] X. A. Zhao, E. D. Guestrin, D. Sayenko, T. Simpson, and M. Gauthier, "Typing with Eye-Gaze and Tooth-Clicks," in ETRA '12 Proceedings of the Symposium on Eye Tracking Research and Applications, 2012, pp. 341-344.

[3] T. P. A. Guillamet, M. T. M. Teixidó, A. F. Viso, and C. R. J. Palacín, "Implementation of a Robust Absolute Virtual Head Mouse Combining Face Detection, Template Matching and Optical Flow Algorithms," Telecommunication Systems, vol. 52, no. 3, pp. 1479-1489, 2013.

[4] Z. Hao and Q. Lei, "Vision-Based Interface : Using Face and Eye Blinking Tracking with Camera," in IITA'08 Second International Symposium on Intelligent Information Technology Applications, 2008.

[5] M. Betke, J. Gips, and P. Fleming, "The Camera Mouse: Visual tracking of body features to provide computer access for people with severe disabilities," IEEE Transactions on Neural Systems and Rehabilitation Engineering, vol. 10, no. 1, pp. 1-10, 2002.

[6] I. S. Mackenzie and B. Ashtiani, "BlinkWrite : Efficient Text Entry using Eye Blinks," Universal Access in the Information Society, vol. 10, no. 1, pp. 69-80, 2011.

[7] P. Biswas and P. Langdon, "A New Interaction Technique Involving Eye Gaze Tracker and Scanning System," in Proceedings of the 2013 Conference on Eye Tracking, 2013, pp. 67-70.

[8] K. Grauman, M. Betke, J. Lombardi, J. Gips, and G. R. Bradski, "Communication via Eye Blinks and Eyebrow Raises : Video-based Human-Computer Interfaces," Universal Access in the Information Society, vol. 2, no. 4, pp. 359-373, 2003.

[9] S. Yang, C. Lin, S. Lin, and C. Lee, "Design of Virtual Keyboard using Blink Control Method for the Severely Disabled," Computer Methods and Programs in Biomedicine, vol. 111, no. 2, pp. 410-418, 2013.

[10] R. Quain and M. M. Khan, "Portable Tongue-Supported Human Computer Interaction System Design and Implementation," in 2014 36th Annual International
Conference of the IEEE Engineering in Medicine and Biology Society (EMBC), 2014, pp. 6302-6307.

[11] M. Kumar, A. Paepcke, and T. Winograd, "EyePoint : Practical Pointing and Selection Using Gaze and Keyboard," in CHI'07 Proceedings of the SIGCHI Conference on Human Factors in Computing Systems, 2007, pp. 421-430.

[12] K. Arai and R. Mardiyanto, "Eye-based HCI with Full Specification of Mouse and Keyboard using Pupil Knowledge in the Gaze Estimation," in Proceedings - 2011 8th International Conference on Information Technology: New Generations, ITNG 2011, 2011, pp. 423-428.

[13] W. Siriluck, S. Kamolphiwong, and T. Kamolphiwong, "Blink and Click," in Proceedings of the 1st International Convention on Rehabilitation Engineering and Assistive Technology: in Conjunction with 1st Tan Tock Seng Hospital Neurorehabilitation Meeting, 2007, pp. 43-46.

[14] A. Huckauf and M. H. Urbina, "Object Selection in Gaze Controlled Systems : What You Don 't Look At Is What You Get," ACM Transactions on Applied Perceptron, vol. 8, no. 2, pp. 1-14, 2011.

[15] J. V. Singh and G. Prasad, "Enhancing an Eye-Tracker based Human-Computer Interface with Multi-modal Accessibility Applied for Text Entry," International Journal of Computer Applications, vol. 130, no. 16, pp. 16-22, 2015.

[16] A. Huckauf, M. H. Urbina, and B. Weimar, "On Object Selection in Gaze Controlled Environments," Journal of Eye Movement Research, vol. 2, no. 4, pp. 1-7, 2008.

[17] Y. Gizatdinova, O. Spakov, and V. Surakka, "Comparison of Video-Based Pointing and Selection Techniques for HandsFree Text Entry," in AVI'12 Proceedings of the International Working Conference Advanced Visual Interfaces, 2012, pp. 132-139.

[18] A. Posusta, A. J. Sporka, O. Polacek, S. Rudolf, and J. Otahal, "Control of Word Processing Environment using Myoelectric Signals," Journal on Multimodal User Interfaces, vol. 9, no. 4, pp. 299-311, 2015.

[19] V. Rantanen, J. Verho, J. Lekkala, O. Tuisku, V. Surakka, and T. Vanhala, "The Effect of Clicking by Smiling on the Accuracy of Head-Mounted Gaze Tracking," in ETRA'12 Proceedings of the Symposium on Eye Tracking Research and Applications, 2012, pp. 345-348. 